\title{
The phase-space density distribution of dark matter halos
}

\author{
Liliya L. R. Williams* \\ Univ. of Minnesota, USA \\ E-mail: llrweastro.umn.edu

\section{Crystal Austin} \\ Univ. of Minnesota, USA \\ E-mail: caustineastro.umn.edu

\section{Eric Barnes} \\ Univ. of Minnesota, USA \\ E-mail: barneseastro.umn.edu
}

\section{Arif Babul}

Univ. of Victoria, B.C., Canada

E-mail: babul@uvic.ca

\section{Julianne Dalcanton}

Univ. of Washington, USA

E-mail: jdeastro.washington.edu

High resolution N-body simulations have all but converged on a common empirical form for the shape of the density profiles of halos, but the full understanding of the underlying physics of halo formation has eluded them so far. We investigate the formation and structure of dark matter halos using analytical and semi-analytical techniques. Our halos are formed via an extended secondary infall model (ESIM); they contain secondary perturbations and hence random tangential and radial motions which affect the halo's evolution at it undergoes shell-crossing and virialization. Even though the density profiles of NFW and ESIM halos are different their phase-space density distributions are the same: $\rho / \sigma^{3} \propto r^{-\alpha}$, with $\alpha=1.875$ over $\sim 3$ decades in radius. We use two approaches to try to explain this "universal" slope: (1) The Jeans equation analysis yields many insights, however, does not answer why $\alpha=1.875$. (2) The secondary infall model of the 1960's and 1970's, augmented by "thermal motions" of particles does predict that halos should have $\alpha=1.875$. However, this relies on assumptions of spherical symmetry and slow accretion. While for ESIM halos these assumptions are justified, they most certainly break down for simulated halos which forms hierarchically. We speculate that our argument may apply to an "on-average" formation scenario of halos within merger-driven numerical simulations, and thereby explain why $\alpha=1.875$ for NFW halos. Thus, $\rho / \sigma^{3} \propto r^{-1.875}$ may be a generic feature of violent relaxation.

Baryons in Dark Matter Halos

5-9 October 2004

Novigrad, Croatia

\footnotetext{
${ }^{*}$ Speaker.
} 


\section{Introduction}

There is a broad consensus that gravity driven evolution of the space distribution of collisionless dark matter, starting from some realistic matter power spectrum results in virialized objects whose spherically averaged density profi le is well represented by the NFW prescription [3] or its close variants [2]. However, why the dark matter profi les have this shape is as yet to be determined. In an effort to shed light on the issue, [4] investigated the phase-space structure of dark matter halos from N-body simulations. They found that the "poor man's" phase-space density, $f(r)=\rho / \sigma^{3}$, is a power law over 3 decades in radius, $f(r) \propto r^{-\alpha}$, with exponent $\alpha=1.875=15 / 8$.

We have developed an alternative method of generating dark matter halos, based on [5]. It is an analytical scheme which treats collapse, shell-crossing and virialization of spherically symmetric halos. The halo contains secondary perturbations whose properties are calculated from the same power spectrum that gives rise to the main halo. These secondary perturbations induce random tangential and radial motions within the halo. The ensuing collapse can be likened to slow accretion of lumpy matter; there are no major mergers in our scheme; [6]. We call these halos ESIM, or Extended Secondary Infall Model halos. Even though ESIM and NFW halos are generated in very different ways, and have different density profi les, their phase-space density distribution, and the value of $\alpha$ is the same for both. Our goal is to understand why is $\alpha=1.875=15 / 8$ for both.

\section{The Jeans equation analysis of phase-space density distribution}

Equilibrium, non-rotating halos with isotropic velocity ellipsoids obey this Jeans equation:

$$
\frac{d}{d r}\left[\frac{-r^{2}}{G \rho} \frac{d\left(\rho \sigma^{2}\right)}{d r}\right]=\frac{d}{d r} M(<r)
$$

Following [4] we use scaled variables, $x=r / r_{0}$, and $y=\rho / \rho_{0}$. We assume power law behavior of $f(r)$ with constant $\alpha$ for any given halo, but allow the density profi le to have changing log-log slope, $y \propto x^{-\beta(x)}$. With these, eq. 2.1 can be rewritten in terms of $x, y$, exponents $\alpha$ and $\beta$, and a normalization constant. Unlike [4] we work with an equation obtained by differentiating eq. 2.1:

$$
15 \beta^{\prime \prime}-3 \beta^{\prime}(8 \alpha-5 \beta-5)=(2 \alpha+\beta-6)(2 \alpha-5 \beta)(2 / 3[\alpha-\beta]+1)
$$

Here, $\beta^{\prime}$ and $\beta^{\prime \prime}$ are derivatives of $\beta$ with respect to $-\ln x$. (Recall that $\beta=-d \ln y / d \ln x$.)

Because eq. 2.2 has many solutions (i.e. halo density profi les), it would help to make analytic inroads into the analysis of its solution space. We do just that: we derive a family of solutions:

$$
\beta^{\prime}=\beta_{m}^{\prime}-2 / 3\left(\beta-\beta_{0}\right)^{2}, \quad \beta_{m}^{\prime}=3(2 \alpha+5)^{2} / 200, \quad \beta_{0}=(14 \alpha+15) / 20 .
$$

All the halos that obey these three equations asymptote to very nearly constant $\beta$ slopes at large and small radii, and have non-zero derivatives of $\beta$ in-between. We will refer to this as the main family of solutions. Each member is completely defi ned by specifying $\alpha$. As far as we can tell, this is the only family that has a closed form analytical description. Note that the 'critical solution' identifi ed by [4] is a member of this family; it is obtained by setting $\alpha=1.875$.

Figure 1(a) is a schematic representation of all solutions of eq. 2.2. A few main family solutions are shown as solid vertical red line segments; the inner density cusp for each is given by 

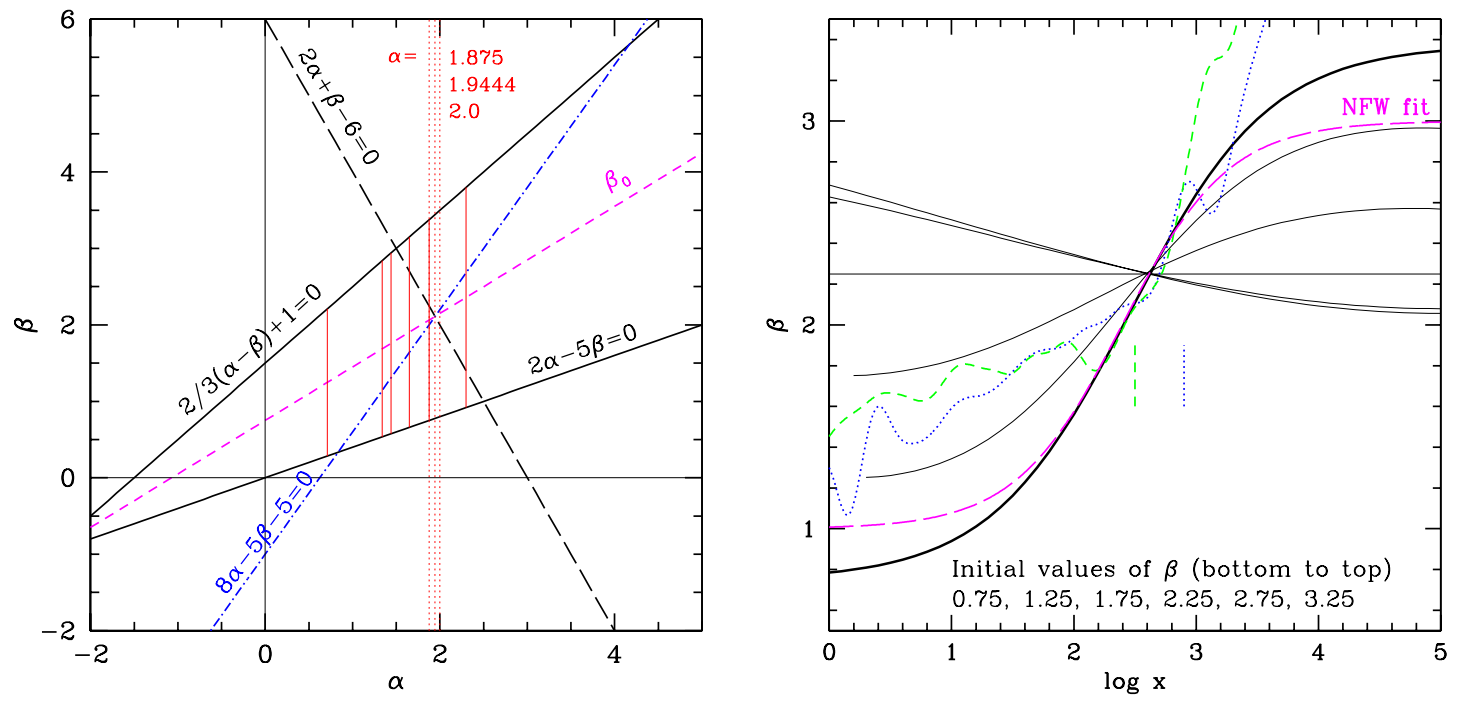

Figure 1: (a) Pictorial depiction of solutions of eq. 2.2. $\alpha=1.875$ is the slope of the phase-space density profiles of NFW and ESIM halos; $\alpha=1.9444$ results in periodic, zero-damping solutions of eq. 2.2. Six solid vertical line segments are examples of the main family of solutions. Labeled lines: factors in eq. 2.2; $\beta_{0}$ is given in eq. 2.3. (b) A set of solutions of eq. 2.2, with constant $\alpha=1.875$ but varying initial values of $\beta=1.25,1.75, \ldots 3.25$; initial $\beta^{\prime}=0$ for all. The thick line belongs to the main family of solutions; note that NFW empirical fit follows this solution closely for intermediate values of $\beta$. Dotted and dashed lines are two ESIM halos; short vertical line segments indicate their virial radii. Normalization of radius $x$ is arbitrary.

$\left(2 \alpha-5 \beta_{\text {in }}\right)=0$, the outer density profi le slope is given by $\left(2 / 3\left[\alpha-\beta_{\text {out }}\right]+1\right)=0$. Fig. 1(a) illustrates the role these factors on the RHS of eq. 2.2 play in defi ning main family solutions.

One of these six vertical line segments is for $\alpha=1.875$; the corresponding $\beta(x)$ is shown as thick solid curve in fi g. 1(b). Other curves are what we call $\alpha$-family solutions, obtained by keeping $\alpha$ constant, but varying initial conditions: $\beta$ and $\beta^{\prime}$. Note that one of the members of the $\alpha$-family is a power law density profi le, $\beta=$ const. The $\beta$ value for the power law solution of an arbitrary $\alpha$-family is obtained by solving $(2 \alpha+\beta-6)=0$; see eq. 2.2 and long-dash line in fi g. 1(a).

Does eq. 2.2 allow any special $\alpha$ values? The equation is a non-linear damped oscillator. For some $\alpha$ the $x$-averaged value of the 'dissipation' term, $-3 \beta^{\prime}(8 \alpha-5 \beta-5)$ is zero. This value is $\alpha=35 / 18 \approx 1.9444$. When $\alpha=35 / 18$ any initial condition for $\beta$ and $\beta^{\prime}$ results in a periodic, constant amplitude function of $\beta$ vs. $x$. Value $\alpha=35 / 15$ is only $\sim 4 \%$ different from $\alpha=1.875$. Aside from this coincidence (or not?), and many other interesting insights provided by the analysis of eq. 2.2, we were not able to identify why $\alpha=1.875$ is special. Next, we take a different, and more promising approach to uncover why $\alpha=1.875$ for ESIM and NFW halos alike.

\section{Secondary infall analysis of phase-space density distribution}

Consider the initial stages of halo collapse in the context of secondary infall models. A small constant central mass excess, $\delta M_{0}$ is surrounded by material of average density. The dynamics of the pre-turn-around period is described by the parametric equations of [1]. The turn-around radius for a shell of initial comoving $r_{i}$, is $r_{m}=r_{i} \bar{\delta}^{-1}$, and $\bar{\delta}$ is the initial fractional overdensity inside $r_{i}$. 
Then, $M\left(<r_{i}\right) \propto \rho r_{i}^{3}$, so $r_{i} \propto M^{1 / 3}$. Also, $\bar{\delta}=\delta M_{0} / M \propto M^{-1}$. Combining these scalings we get $r_{m} \propto M^{4 / 3}$. After reaching turn-around each shell collapses back a little; one typically assumes a constant collapse factor. Assume that each shell spends most of the time at its apocenter, and so most of its mass is located at that radius, which is $\propto r_{m}$. The resulting density distribution in the proto-halo is, $\rho(r) \propto r^{-2} d M / d r$, or, $\rho \propto M^{-3} \propto r_{m}^{-9 / 4}$. This is a well known result.

In the real Universe the collapse of material will not be purely radial; there will be some random motion of particles, and associated kinetic energy. We speculate that during the early stages of collapse the kinetic energy will be derived from the gravitational potential energy, and therefore will be proportional to the potential energy: $\frac{1}{2} v^{2} \propto G M / r_{m}$. Kinetic energy of random motion gives an estimate of the velocity dispersion: $\sigma \propto\left[M / r_{m}\right]^{1 / 2} \propto r_{m}^{-1 / 8}$. So, $\sigma^{3} \propto r_{m}^{-3 / 8}$. Combining $\rho(r)$ from the previous paragraph with $\sigma(r)$, we get $\rho / \sigma^{3} \propto\left(r^{-9 / 4}\right) /\left(r^{-3 / 8}\right) \propto r^{-15 / 8}$, i.e. $\alpha=1.875$ !

The result obtained above says that the phase-space density is a function of $r$. Because $E \propto G M / r, E$ is a monotonic function of $r$, at least for systems that are, on average, spherically symmetric. In equilibrium, the total energy $E$ of a particle is its integral of motion. If the halo collapse proceeds slowly then the halo passes through a series of quasi-equilibrium stages. Maybe we can assume that energy is an 'approximate' integral of motion in a slowly collapsing galaxy. In that case, our relation $\rho / \sigma^{3} \propto r^{-1.875}$ can be interpreted as saying that the phase-space density $\left(\rho / \sigma^{3}\right)$ is a function of $E$ only, in compliance with Jeans Theorem.

If dark matter is collisionless, then the collapse will preserve the phase-space density as calculated above. So the fi nal virialized dark matter halos, whatever their density and velocity dispersion profi les, will have the same phase-space density distribution that was characteristic of the early stages of collapse. Therefore, virialized halos are expected to have $\rho / \sigma^{3} \propto r^{-1.875}$. This argument relies on many approximations, most of which can be justifi ed for spherically symmetric, smoothly accreting ESIM halos. However, one is hard pressed to see why these approximations would hold in a hierarchical formation model. We speculate that the argument could apply to an 'average' situation; after all, NFW is an average shape of numerically generated halos.

Finally, we note the connection between the above argument and the $\alpha$-family solutions of $\S 2$. The various evolutionary stages of halos have different density profi les. We claim that all these are represented by members of the same $\alpha=1.875$-family. The earliest epoch halos have power law density profi les with $\beta=9 / 4$, value derived at the top of this $\S$; the same value is derived using Jeans equation analysis - the horizontal line in fi g. 1(b). Later stages of halo evolution develop changing slope density profi les, represented by more and more curved lines in fi g. 1(b). The fi nal stage, the thick solid line is a good approximation to the NFW profi le obtained from simulations.

\section{References}

[1] Gunn, J.E. \& Gott, J.R. 1972, ApJ, 176, 1

[2] Moore, B., Governato F., Quinn, T., Stadel, J. \& Lake, G. 1998, ApJ, 499, L5

[3] Navarro, J.F., Frenk, C.S. \& White, S.D.M. 1997, ApJ, 490, 493

[4] Taylor, J.E. \& Navarro, J.F. 2001, ApJ, 563, 483

[5] Ryden, B.S. \& Gunn, J.E. 1987, ApJ, 318, 15

[6] Williams, L.L.R., Babul, A. \& Dalcanton, J.J. 2004, ApJ, 604, 18 